\title{
Integrating Unmanned Aerial Vehicle and Deep Learning Algorithm for Pipeline Monitoring and Inspection in the Oil and Gas Sector
}

\author{
Uchechi F. Ukaegbu \\ Department of Mechanical \& Industrial Engineering \\ University of Johannesburg \\ Johannesburg, South Africa \\ ukaegbuuchechi531@gmail.com \\ Modestus O. Okwu \\ Department of Mechanical \& Industrial Engineering \\ Technology, University of Johannesburg \\ Johannesburg, South Africa \\ okwu.okechukwu@fupre.edu.ng
}

\begin{abstract}
Organizations invest heavily during the installation of pipeline facilities which spans across extensive geographical linkage. Also, the issue of pipeline protection and safety must be taken into consideration to have a free flow of oil and gas at inbound and outbound locations. This has necessitated the need for routine inspection and monitoring of pipelines for structural integrity, continued safe operations, and also to monitor intruders/potential trespassers at such locations. This research is focused on the elucidation of a modular unmanned aerial vehicle (UAV) integrated with deep learning algorithms for monitoring and security response to obtain very useful information around the oil and gas facility. The deep learning model employed in this study had training, validation and testing accuracies of $88.3 \%, 87.5 \%$, and $83.3 \%$ respectively. Also, training and validation losses of 0.3583 and 0.3649 were obtained. The suggested integrated UAV has a low maintenance requirement, high endurance, and is costeffective. It has a superior advantage over the manned aerial vehicles (MAV) currently in use since safety is greatly improved, the cost is reduced, adequate information is obtained and communication is enhanced.
\end{abstract}

CCS Concepts: • Computing methodologies $\rightarrow$ Artificial Intelligence; Computer vision.

Permission to make digital or hard copies of all or part of this work for personal or classroom use is granted without fee provided that copies are not made or distributed for profit or commercial advantage and that copies bear this notice and the full citation on the first page. Copyrights for components of this work owned by others than ACM must be honored. Abstracting with credit is permitted. To copy otherwise, or republish, to post on servers or to redistribute to lists, requires prior specific permission and/or a fee. Request permissions from permissions@acm.org.

icARTi '21, December 9-10, 2021, Virtual Event, Mauritius

(c) 2021 Association for Computing Machinery.

ACM ISBN 978-1-4503-8575-6/21/12 .. \$15.00

https://doi.org/10.1145/3487923.3487924

\author{
Lagouge K. Tartibu \\ Department of Mechanical \& Industrial Engineering \\ Technology, University of Johannesburg \\ Johannesburg, South Africa \\ Itatibu@uj.ac.za \\ Isaac O. Olayode \\ Department of Mechanical \& Industrial Engineering \\ Technology, University of Johannesburg \\ Johannesburg, South Africa \\ olayode89@gmail.com
}

Keywords: Unmanned aerial vehicle, Deep learning algorithm, Pipeline, Monitoring, Inspection

\section{ACM Reference Format:}

Uchechi F. Ukaegbu, Lagouge K. Tartibu, Modestus O. Okwu, and Isaac O. Olayode. 2021. Integrating Unmanned Aerial Vehicle and Deep Learning Algorithm for Pipeline Monitoring and Inspection in the Oil and Gas Sector. In International Conference on Artificial Intelligence and its Applications (icARTi '21), December 9-10, 2021, Virtual Event, Mauritius. ACM, New York, NY, USA, 6 pages. https: //doi.org/10.1145/3487923.3487924

\section{Introduction}

Pipelines are very useful materials designed to transport materials in the form of gases, liquid, crude oil, sewage, and others, from one location to another. In the oil and gas sector, they are used for transporting crude. The use of pipelines as a means of transporting liquid and gaseous materials started long ago via traditional means and gradual improvement continued till this present time. In time past, bamboo trees were cut down to produce pipeline materials which gradually upgraded to the use of concrete, steel materials, and plastic materials [10]. As a result of long distances covered when mounting pipelines, it is almost impossible for man to monitor the range of pipeline layouts located at different routes and districts since they are channeled or crisscrossed from source through a long range of kilometers to remote locations which could be inaccessible to humans. To protect the resources which are transported via pipelines from one location to another, pre-emptive and regular inspections are required from the source of the material to the destination where the resources are being transported, especially when the material being transported are flammable like crude. If adequate inspection is not maintained, there is a tendency of causing disaster to humans and the environment. In a situation where there is leakage of pipe conveying crude or 
pipe vandalization by intruders, there is a high tendency of an explosion, which is disastrous and can affect living organisms, cause environmental pollution, lead to a stoppage in the delivery process, and could lead to great financial loss [10]. For this reason, oil and gas industries, pipeline industries, and other systems involved in the delivery process that require pipelines as means of transportation adopt several methods to protect pipelines from source to destination. The traditional techniques often used have not yielded much result and company managers keep seeking an approach to monitor pipelines from intruders, leakages, and bursts.

In this era of the fourth industrial revolution, companies are encouraged to deploy autonomous devices or robots for information gathering ranging from leak detection in form of images to operational monitoring of facilities at various locations. Effective path planning is necessary to achieve such. A robot with a manipulator can be stationed to cover long-range pipelines via effective path planning on-site $[2,15]$. Robots can endure and carry out the task efficiently and productively, with a high level of accuracy or resilience than humans, and can also operate in a murky, unsafe, and hazardous environment [26]. Intermittent monitoring and maintenance of the pipeline are required in the oil and gas sector to ensure there is no form of leakage and to guarantee constant and continuous flow of crude. Rather than using the manned aerial vehicle which has several disadvantages, the UAVs are capable of providing a picture-perfect aerial view of any form of leakage and finding a lasting solution to the problem at the early stage.

\section{Manned Aerial Vehicles and Unmanned Aerial Vehicles}

\subsection{Manned Aerial Vehicle}

Manned Aerial Vehicles (MAV), are generally helicopters that are operated by professional pilots especially in the oil and gas sector, military operations, and medical services. Most companies use these vehicles to convey staff and useful materials from one station to another. In the oil and gas sector, MAVs are used to transport workers to offshore and deep-sea sites while some companies use MAVs for security purposes or to provide first aid to staff in challenging situations, others use them for information gathering and security purposes via taking pictures and supplying information to control rooms. Barmpounakis et al [4] contended that MAVs are quite expensive in terms of operational and maintenance cost, also for the fact that they convey humans and expensive equipment to various destinations, there is high risk involved during the navigational process. They could be subject to attack or operational failure. It is, therefore, necessary to look beyond the MAVs.

\subsection{Unmanned Aerial Vehicle}

Unmanned aerial vehicles are flying robots that are programmed to function autonomously. They are equally referred to as drones or flying vehicles without a pilot, capable of navigating autonomously from source to destination using technologies like artificial intelligence, computer vision, obstacle avoidance technologies and others [14]. In other words, unmanned aerial vehicles can be defined as reusable, autonomous, or power-driven vehicles capable of navigation using a remote control or without the assistance of a human pilot [5, 27].

Recently, researchers are paying much more attention to autonomous flying robots (AFR) or unmanned aerial vehicles (UAVs) for surveillance, alternative and faster ways of moving items from source to destinations especially for humanitarian logistics and military operations [14]. A deep description of the application of UAV for effective delivery of items from source to destinations in the era of the fourth industrial revolution was further revealed in ref.[14]. Application of robots in onshore and offshore oil and gas are available elsewhere [17, 20]. Static cameras (SC), MAVs, and UAVs can be used for monitoring and surveillance in the oil and gas sector. A Comparison of SCs with UAV and MAV from the perspective of emergency response has been presented by the author in ref. [4]. According to the observation made, the location of static cameras to capture a particular system could not provide a clear picture as expected. The comparative analysis favours the UAV system in terms of operational use, cost of purchase, energy efficiency, maintenance, and others. This research is focused on the exposition of the UAV embedded with a deep learning algorithm, for possible applications in the oil and gas sector for pipeline monitoring and inspection.

\section{Deep Learning Algorithm}

Deep learning (DL) is a subcategory of machine learning (ML) which is made up of several processing layers, capable of transforming and learning data representation with innumerable classified features [9]. DL is structured in a unified way and consists of three layers which are the input, several hidden layers, and the output layers. Each layer is made up of nodes that are connected to corresponding nodes in successive layers thereby mimicking the structure of the neuron contained in the human brain [11].

A close observation of the DL pattern reveals an input weighted sum which is transmuted by the activation function (AF) to generate outputs in a non-linear form which are fed as input to the end-to-end units of successive layers till the output layer is attained [19]. The iteration process of the forward and backward propagation is required to obtain improved and acceptable weights and biases as the optimal or near-optimal solution. Deep Learning has been applied to solve real-life problems in diverse areas of research studies 
for analysis of image [3], processing of natural language [7], natural disaster prediction [13], the prognosis of cancer [12], nutrient deficiencies detection [23]. DL can be classified into several sub-divisions however, just one of them which is the convolutional neural network (CNN) would be applied in this study. The CNN is widely used for precision agriculture and computer vision applications involving image recognition, classification, and detection due to its sparse interaction, equivalent representation as well as weight capabilities [18].

\section{Related Works}

The authors in ref. [8] combined deep learning technique with the Otsu algorithm on an unmanned aerial vehicle for the detection of oil spillage. Their experiment showed that oil spill was detected effectively and proved to cost effective unlike when satellites systems are being used. The authors recommended that the oil spill images be collated over a long period of time in order to further improve the efficiency of the model. Also, they suggested that the infrared camera should also be incorporated on the UAV for night inspections.

The authors in ref. [28] carried out a review on the use of robots such as remotely operated vehicles, autonomous underwater vehicles, unmanned aerial vehicles and unmanned ground vehicles for inspection in oil and gas facilities. The authors revealed that the UAVs and AUVs has prospects especially with a formidable combination of the visual simultaneous localization and mapping algorithms with deep learning techniques.

The authors in ref. [1] presented a review of several techniques used for the monitoring of oil and gas pipelines for leakages and other defects. They highlighted the advent of drones used as a tool for the inspection and also discussed the pros and cons of various leakage detection techniques.

\section{Materials and Methodology}

\subsection{Acquisition of Dataset/ Training of the CNN Model}

The datasets employed in this study was obtained from a publicly available source [22] and the Nigerian Liquefied Natural Gas (NLNG) research department. It comprises images of oil pipelines, pipelines with leakages, humans not wearing personal protective equipment (PPE), humans wearing PPE that tallies to about 204 images.

The datasets were divided in a ratio of 7:2:1 into training, validation, and test data. They were used to train a ResNet50 model, through transfer learning, for 5 epochs on top of the TensorFlow framework using the google colaboratory with an input shape of $224 \times 224 \times 3$ and a batch size of 10 . The trained model was thereafter converted to a TensorFlow format and optimized for latency and size to improve inference time as recommended by the authors in [24].

\subsection{Embedded System Employed}

The raspberry pi 3B was the embedded system used to interpret and actuate the deep learning algorithms deployed on it. It is made up of a quad-core 64-bit ARM core @1.2GHz, 1GB RAM, 4 USB 2.0 ports, HDMI ports, camera serial interface, display serial interface, micro-USB power port, 40 GPIO pins, Ethernet port, and SD card slot. It uses the Raspbian operating system. The Adam optimizer, categorical cross-entropy loss, and accuracy metric were employed during the compilation of the model. The TensorFlow and OpenCV libraries were installed on the raspberry pi and thereafter, the trained model in TensorFlow lite format was transferred to it.

5.2.1 Setting up the Embedded System for Deep Learning Application. The raspberry pi setup comprises a $2.5 \mathrm{~A}$ $5.1 \mathrm{~V}$ micro-USB power supply, an SD card ranging from 16 to $32 \mathrm{~GB}$, Keyboard and mouse, an HDMI cable, a pi camera or USB camera, a computer or television screen. Thereafter the Raspbian operating system is installed using the New out of Box (NOOBS) installation manager. Also, the TensorFlow and OpenCV libraries were installed in a virtual environment created on the raspberry pi. The trained model from the PC was transferred to the raspberry pi using the FileZilla client software.

\subsection{Materials and Calculations for the Proposed UAV}

The proposed UAV to be used in this study has been previously been developed by the authors for agricultural weed detection and the selective spray of herbicides [25]. However, due to the diverse application of the UAV, its function would be modified for inspection and monitoring purposes in the oil and gas sector. The UAV was built from a readily available quadcopter kit and some calculations were performed to check for the component compatibility before purchasing the kit.

5.3.1 ESC Calculation. The current rating of the ESC is designed to be $20-50 \%$ more than the current rating of the electric motor.

$$
\begin{aligned}
& \text { min ESC amperage }=1.2 \times \text { min amp rating of a motor } \\
& \text { max ESC amperage }=1.5 \times \text { max amp rating of motor }
\end{aligned}
$$

\subsubsection{Battery Calculation.}

$$
\text { Discharge current }=\text { battery capacity } \times \text { C rating }
$$

The higher the rate of current discharge rate, the higher the capacity of the battery to withstand overheating. Also, the ESC amperage rating should not exceed the battery discharge current.

max current drawn $=$ no. of motors $\times$ max current

by the motors drawn by 1 motor 
5.3.3 Thrust Calculation. In order to achieve lift off the ground, the total thrust to total weight ratio should be greater than 1 .

$$
\text { Thrust provided }=\frac{2}{4} \times \text { total weight }
$$$$
\text { by each motor of quadcopter }
$$

$$
\text { Thrust provided by the propellers }=\frac{\pi \times D^{2} \times v \times \Delta v \times p}{4}
$$

Where $\mathrm{D}=$ diameter of propeller

$\mathrm{v}=$ velocity of air

$\Delta \mathrm{v}=$ velocity of accelerated air;

with the assumption that it equals $11.61 \mathrm{~m} / \mathrm{s}$ at $78 \%$ ideal efficiency

$\mathrm{p}=$ density of air

For the electronic speed controller (ESC), its amperage rating was $50 \%$ greater than that of the electric motor. Also, the lithium-polymer battery chosen had a discharge current rate that was higher than the ESCs' amperage ratings to withstand overheating. Also, the calculated thrust provided by the propellers was about twice the weight of the UAV hence lift was assured. The specifications of the UAV's component are shown in Table 1 and Fig 1 displays its design.

\begin{tabular}{|c|c|c|}
\hline $\mathrm{S} / \mathrm{N}$ & Name of Component & Specification \\
\hline 1 & $\begin{array}{l}\text { XXD A2212 brushless } \\
\text { outrunner electric motor }\end{array}$ & $\begin{array}{l}\mathrm{kV}: 1000 \\
\text { Current rating: } 12 \mathrm{~A} / 60 \mathrm{~s} \\
\text { Weight: } 47 \mathrm{~g}\end{array}$ \\
\hline 2 & $\begin{array}{l}\text { Electronic speed con- } \\
\text { troller }\end{array}$ & $\begin{array}{l}\text { Continuous current: } 30 \mathrm{~A} \\
\text { Burst current: } 40 \mathrm{~A}\end{array}$ \\
\hline 3 & APM 2.8 flight controller & $\begin{array}{l}\text { Made up of 3- axis } \\
\text { gyro, accelerometer, and } \\
\text { barometer. }\end{array}$ \\
\hline 4 & Propeller & $\begin{array}{l}\text { Diameter: } 10^{\prime \prime} \\
\text { Pitch: } 4.5^{\prime \prime}\end{array}$ \\
\hline 5 & $\begin{array}{l}\text { ZOP Power } 11.1 \mathrm{~V} 3 \mathrm{~S} \\
\text { lithium-polymer battery }\end{array}$ & $\begin{array}{ll}\text { Capacity: } & \text { 5500MAH } \\
\text { Continuous } & \text { discharge } \\
\text { rate: } 45 \mathrm{C} & \end{array}$ \\
\hline
\end{tabular}

Table 1. Specifications of Selected UAV Components

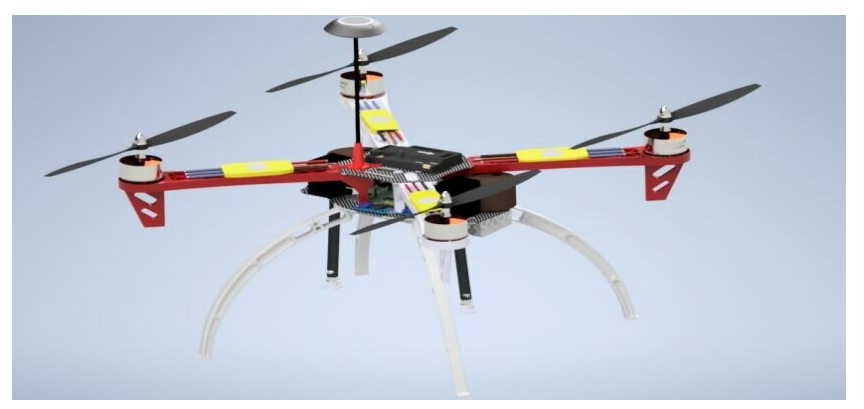

Figure 1. CAD Design of the Proposed UAV

\subsection{Application Procedure- UAV and DL in the Oil and Gas Sector}

Before any flight operation, the following check should be carried out: Inspect the frame for any physical damage or crack; Inspect the motors and propellers for damage or presence of debris and ensure that both are secured correctly; Inspect all electrical components for correct functioning. Inspect the installation of parts that are removable such as the battery. The UAV system is designed to inspect and monitor pipeline facilities for leakages and pipeline vandalization by intruders. It would operate in such a way that as the UAV moves via the route of the pipeline, the deployed CNN model would examine pictures taken by the picamera of the raspberry pi mounted on the UAV. When a pipeline leakage and/or human not wearing a PPE is within the picture frame, GPIO 18 of the raspberry pi would be activated to supply $3.3 \mathrm{~V}$. This voltage, together with the VCC from the raspberry pi, would serve as inputs to turn on a relay. The output from the relay could be used to send information to the operator at the ground station for necessary actions. This information may be in form of image data, short message service (SMS), etc. and it would display the problem detected as well as the GPS location of the UAV at that particular point in time. A voltage source could be connected to the 'common' and a GSM module (already programmed) connected to the 'normally open' of the relay such that when it clicks on, the GSM module will be powered on to send a short message service (SMS) notification. However, if no pipe leakage and/or thirdparty interference is spotted, the UAV would continue in its pre-planned flight path. Effective path planning algorithms would be employed to ensure that the UAV avoids obstacles during its operation.

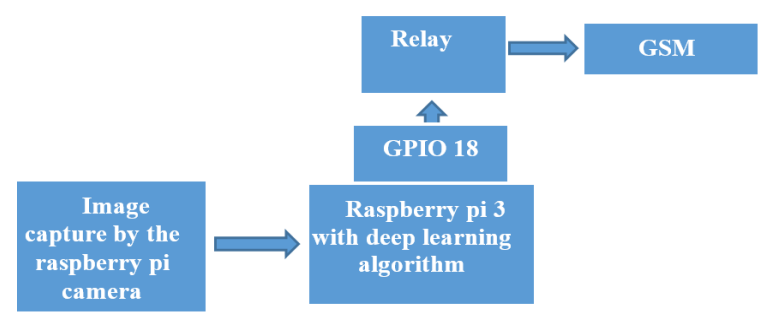

Figure 2. A Flowchart showing the proposed methodology

\section{Results and Analysis}

The trained CNN model generated 8196 trainable parameters from the 204 trained images. Training, validation, and test accuracies of $88.3 \%, 87.5 \%$, and $83.3 \%$ respectively were collated. Furthermore, training and validation losses of 0.3583 and 0.3649 were obtained respectively. From the results obtained, there was no observed over-fitting as the difference between the training and validation accuracies was minimal (about 0.8\%). Fig 3 and Fig 4 show the accuracy and loss graphs. 


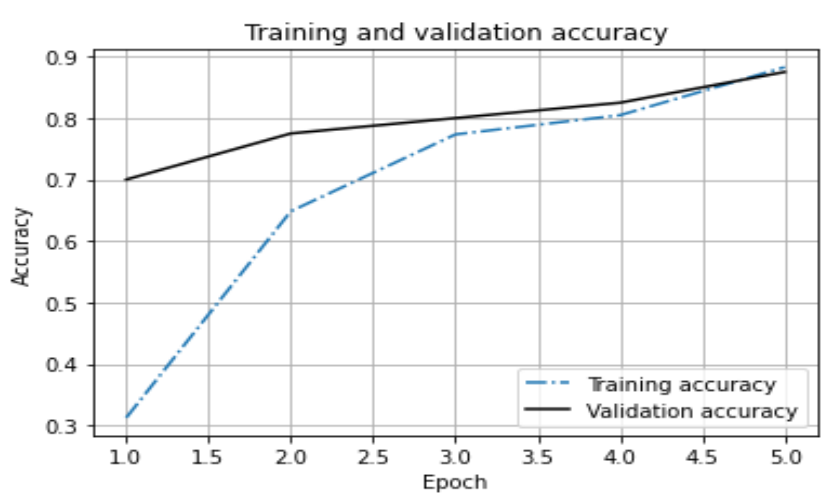

Figure 3. Accuracy vs Epoch

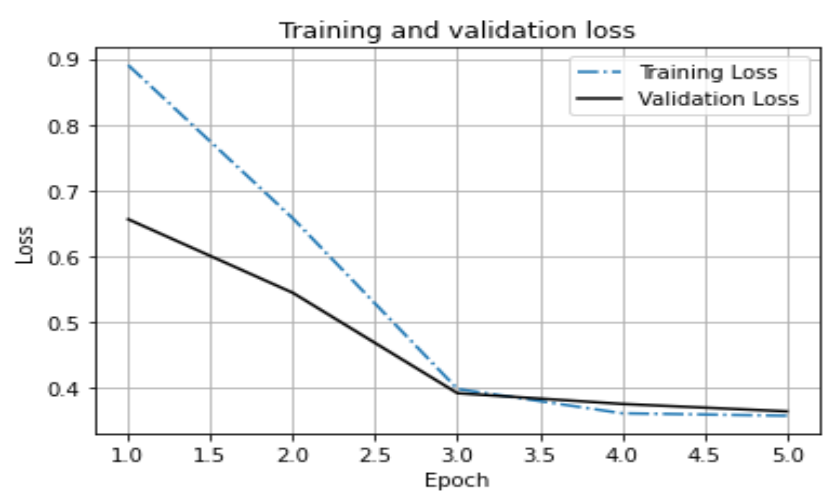

Figure 4. Loss vs Epoch

The confusion matrix, shown in Fig 5, revealed that some images of 'pipelines' were misclassified as 'PPE'(humans wearing PPE) and this is attributed to the fact that some images contain human operators wearing PPE standing close to the pipelines. This would however not affect our application procedure, as there was no misclassification recorded for 'pipe_leakages' and 'not_PPE' (humans not wearing PPE).

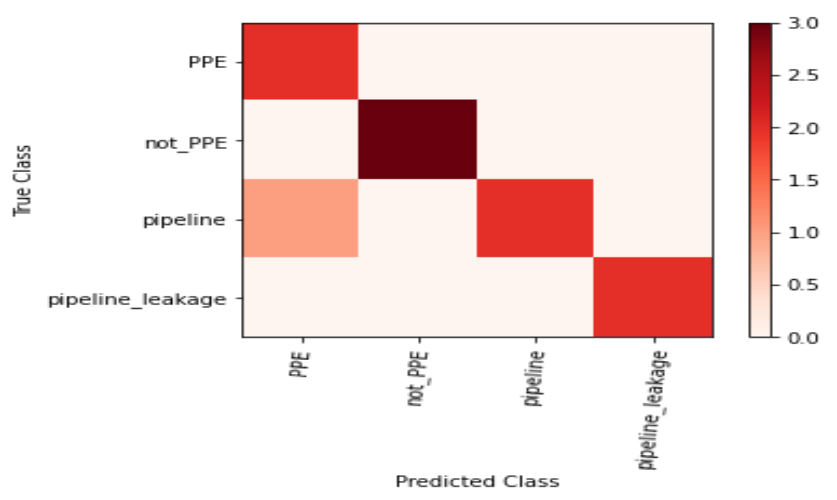

Figure 5. Confusion Matrix for the CNN Model

\section{Conclusion}

This study provides an overview about how UAVs could be integrated with DL for monitoring and inspection purposes in the oil and gas sector. A CNN model was developed to spot out pipe leakages and/or third-party interference that could be deployed on an embedded system and incorporated on a UAV for sending notifications to the ground station. Although the CNN model trained is suitable for this application, an increase in the number of training images would significantly improve the accuracy of the model. Finally, a prospect of this work would involve sending image data immediately after a pipe leakage or intruder is detected by the UAV courtesy of the DL algorithm.

\section{References}

[1] Khalid Ghanim Aljuaid, Mohammad Abdulwahab Albuoderman, Emad Abdullah AlAhmadi, and Jamshed Iqbal. 2020. Comparative Review of Pipelines Monitoring and Leakage Detection Techniques. In 2020 2nd International Conference on Computer and Information Sciences (ICCIS). IEEE, $1-6$.

[2] George A Antaki. 2003. Piping and pipeline engineering: design, construction, maintenance, integrity, and repair. CRC Press.

[3] Szilárd Aradi. 2020. Survey of deep reinforcement learning for motion planning of autonomous vehicles. IEEE Transactions on Intelligent Transportation Systems (2020).

[4] Emmanouil N Barmpounakis, Eleni I Vlahogianni, and John C Golias. 2016. Unmanned Aerial Aircraft Systems for transportation engineering: Current practice and future challenges. International fournal of Transportation Science and Technology 5, 3 (2016), 111-122.

[5] HaiYang Chao, YongCan Cao, and YangQuan Chen. 2010. Autopilots for small unmanned aerial vehicles: a survey. International fournal of Control, Automation and Systems 8, 1 (2010), 36-44.

[6] Olson P. G. and Muff S. H. 2017. Using drones offshore. https: //www.njordlaw.com/knowledge/using-drones-offshore.

[7] A Hassan and A Mahmood. 2018. Convolutional recurrent deep learning model for sentence classification. IEEE Access 6: 13949-13957.

[8] Zeyu Jiao, Guozhu Jia, and Yingjie Cai. 2019. A new approach to oil spill detection that combines deep learning with unmanned aerial vehicles. Computers \& Industrial Engineering 135 (2019), 1300-1311.

[9] Andreas Kamilaris and Francesc X Prenafeta-Boldú. 2018. Deep learning in agriculture: A survey. Computers and electronics in agriculture 147 (2018), 70-90.

[10] Henry Liu. 2019. Revival: Pipeline Engineering (2004). Taylor \& Francis Group. https://books.google.com.pk/books?id=ljZRuAEACAAJ

[11] Gary Marcus. 2018. Deep learning: A critical appraisal. arXiv preprint arXiv:1801.00631 (2018).

[12] Ghulam Murtaza, Liyana Shuib, Ainuddin Wahid Abdul Wahab, Ghulam Mujtaba, Henry Friday Nweke, Mohammed Ali Al-garadi, Fariha Zulfiqar, Ghulam Raza, and Nor Aniza Azmi. 2020. Deep learningbased breast cancer classification through medical imaging modalities: state of the art and research challenges. Artificial Intelligence Review 53, 3 (2020), 1655-1720.

[13] Sella Nevo, Vova Anisimov, Gal Elidan, Ran El-Yaniv, Pete Giencke, Yotam Gigi, Avinatan Hassidim, Zach Moshe, Mor Schlesinger, Guy Shalev, et al. 2019. ML for flood forecasting at scale. arXiv preprint arXiv:1901.09583 (2019).

[14] Modestus Okwu, Lagouge Tartibu, and Mosa Machesa. 2021. Fourth Industrial Revolution and Sustainable Impact in Autonomous Fleets for Effective Supply Chain Network in Manufacturing Systems.

[15] Modestus O Okwu, Oluwayomi J Oyejide, Lagouge K Tartibu, and Mosa Machesa. [n.d.]. DEVELOPMENT OF A MODULAR PICK AND 


\section{PLACE ROBOT/AUTOMATED GUIDED VEHICLE (AGV). ([n. d.]).}

[16] Penn State Extension. 2018. Using Drone Technology in the Oil and Gas Field. https://extension.psu.edu/using-drone-technology-in-theoil-and-gas-field

[17] Phillips. 2017. CyPhy Works and Pilot Thomas Logistics Partner to Deliver UAV Services to the Oil and Gas Industry. https://dronelife.com/cyphy-works-pilot-thomas-logisticspartner-deliver-UAV-services-oil-ga@s-industry/

[18] Samira Pouyanfar, Shu-Ching Chen, and Mei-Ling Shyu. 2017. An efficient deep residual-inception network for multimedia classification. In 2017 IEEE International Conference on Multimedia and Expo (ICME). IEEE, 373-378.

[19] Tausifa Jan Saleem and Mohammad Ahsan Chishti. 2019. Deep learning for Internet of Things data analytics. Procedia computer science 163 (2019), 381-390.

[20] Amit Shukla and Hamad Karki. 2016. Application of robotics in offshore oil and gas industry-A review Part II. Robotics and Autonomous Systems 75 (2016), 508-524.

[21] Amit Shukla and Hamad Karki. 2016. Application of robotics in onshore oil and gas industry-A review Part I. Robotics and Autonomous Systems 75 (2016), 490-507.

[22] Shutterstock. 2021. https://www.shutterstock.com/search/pipeline+ leaks?page $=3$.
[23] Uchechi Ukaegbu, Lagouge Tartibu, Timothy Laseinde, Modestus Okwu, and Isaac Olayode. 2020. A deep learning algorithm for detection of potassium deficiency in a red grapevine and spraying actuation using a raspberry pi3. In 2020 International Conference on Artificial Intelligence, Big Data, Computing and Data Communication Systems (icABCD). IEEE, 1-6.

[24] Uchechi Ukaegbu, Lagouge Tartibu, and Modestus Okwu. 2020. DEEP LEARNING HARDWARE ACCELERATORS FOR HIGH PERFORMANCE IN SMART AGRICULTURAL SYSTEMS: AN OVERVIEW.

[25] Uchechi F Ukaegbu, Lagouge K Tartibu, Modestus O Okwu, and Isaac O Olayode. 2021. Development of a Light-Weight Unmanned Aerial Vehicle for Precision Agriculture. Sensors 21, 13 (2021), 4417.

[26] Wikipedia contributors. 2021. Robotics - Wikipedia, The Free Encyclopedia. https:/en.wikipedia.org/w/index.php?title=Robotics\& oldid=1039356384 [Online; accessed 22-March-2021].

[27] Rafael Yanushevsky. 2011. Guidance of unmanned aerial vehicles. CRC press.

[28] Leijian Yu, Erfu Yang, Peng Ren, Cai Luo, Gordon Dobie, Dongbing $\mathrm{Gu}$, and Xiutian Yan. 2019. Inspection robots in oil and gas industry: a review of current solutions and future trends. In 201925 th International Conference on Automation and Computing (ICAC). IEEE, 1-6. 